\title{
Los recursos tecnológicos y la reconfiguración de la educación médica
}

\section{Technological Resources and the Reconfiguration of Medical Education}

\author{
Juan Manuel Muñoz Cano \\ Universidad Juárez Autónoma de Tabasco \\ juan.munoz@ujat.mx \\ México
}

Teresita del Niño Jesús Maldonado Zalazar
Universidad Pedagógica Nacional, unidad 049
petite_thereses_rouge@hotmail.com

México

\begin{abstract}
Resumen:
La necesidad del trabajo escolar en la educación médica en ambientes virtuales a causa de la pandemia mostró las deficiencias en el currículo, la pedagogía y el uso de los recursos tecnológicos. Se hizo un estudio cualitativo, descriptivo, con enfoque hermenéutico. Se revisó la construcción del modelo de educación médica de Flexner que data de 1910, así como de los determinantes que lo hacen persistir. El análisis se hizo desde tres enfoques para la aplicación de los recursos tecnológicos: el trabajo en el aula, el currículo y la educación en valores. Los análisis mostraron las causas de la petrificación del modelo de currículo, que emerge del positivismo y de la modernidad. Se revisaron los efectos que en el modelo han tenido las tendencias del neopositivismo y la posmodernidad. Se explica la disfuncionalidad del proceso escolar mediante el modelo del estructuralismo funcionalista. Esto muestra cómo se ha obstaculizado la transición a los enfoques educativos tendientes a resolver problemas de la vida real con auxilio de los recursos tecnológicos. Se necesitan modificaciones de forma y fondo para que los recursos tecnológicos no sean solo un medio para llevar el modelo actual a lo virtual.
\end{abstract}

Palabras clave: B-learning, flexibilidad curricular, currículo integrado, aprendizaje basado en problemas, preguntas clínicamente estructuradas.

\begin{abstract}
:
The need for schoolwork in virtual settings because of the pandemic showed deficiencies in curriculum, pedagogy, and the use of technological resources. A qualitative, descriptive, hermeneutical study was conducted. The construction of Flexner's medical education model, dating back to 1910, as well as the determinants that make it persist, were revised. The analysis was made from three approaches to the application of technological resources: classroom work, curriculum, and value education. The analysis showed the causes of the petrification of the curriculum model, which emerges from positivism and modernity. The effects of neopositivist and postmodernity on the model were reviewed as well. The dysfunctionality of the school process is explained by the model of functionalist structuralism. This shows how the transition to educational approaches to solving real-life problems has been hampered with the help of technological resources. Substantive modifications of form and content are needed so that technological resources are not just a means to bring the current model to the virtual.
\end{abstract}

Keywords: B-learning, Curricular flexibility, Integrated curriculum, Problem-based learning, Clinically structured questions.

Recibido: 07/07/2020 | Aceptado: 24/08/2020 | Publicado: 08/01/2021

| pág. 1 - 30 


\section{Los recursos tecnológicos y la reconfiguración de la educación médica}

\section{Introducción}

La estructura de una institución de educación médica debe reconfigurarse en función de las necesidades contextuales que se analizan a partir de datos epidemiológicos y de las ciencias que sustentan las nuevas formas de desarrollar las actividades escolares y el aprendizaje. Las problemáticas socioeconómicas y en salud pública actuales son la pandemia de Enfermedades No Transmisibles (ENT) asociadas a la nutrición, y la crisis global de pobreza y pobreza extrema, que ya se extiende a 40 años, resultado de la imposición del neoliberalismo como versión dominante del capitalismo (De Souza, 2020, p. 9). Estas problemáticas se producen por efectos de las actividades de explotación del planeta a causa de las prácticas del neoliberalismo que se traducen en el calentamiento global, efecto primordial del Antropoceno (Willet et al., 2019). Sobre este ambiente se produjo una pandemia a causa de la infección con el virus COVID-19, con elevaciones súbitas de incidencia con alta carga de mortalidad en personas con riesgos relacionados con enfermedades asociadas a la alimentación, aunque la letalidad general es relativamente baja. Esta es una situación histórica, y como tal ha de tratarse desde la perspectiva de la historia como ciencia que responde a una pregunta: ¿por qué los sucesos ocurrieron así y no de otra manera? (Collingwood, 2004).

La pandemia de ENT es causada primordialmente por la alta ingestión de alimentos ultra procesados (Tabung et al., 2017), disponibles sobre todo entre las clases desfavorecidas de México. Estas enfermedades son hipertensión, diabetes mellitus de tipo 2 (DM2), obesidad, enfermedad cardiovascular y enfermedad renal. Así, DM2 es la primera causa de muerte en mujeres y la segunda en hombres desde hace varios lustros, y 
se contabilizaron 83,000 defunciones por esta causa en 2010 (INSP, 2020). La hipertensión arterial, de acuerdo con los nuevos criterios de 2017 de la American College of Cardiology/ American Heart Association, se calcula que la padece $60 \%$ de la población mexicana mayor de 20 años (Rosas \& Borrayo, 2018). A esto se adiciona la crisis de pobreza, pues la proporción de población de México en estas condiciones en 2018 fue $41.9 \%$, y de pobreza extrema, 7.4\% (CONEVAL, 2020).

Para afrontar de mejor manera estos problemas es necesario reconfigurar los procesos educativos. De manera primordial, entre ellos, la educación médica. Las necesidades de modificaciones con el auxilio de los recursos tecnológicos se colocan en tres ámbitos primordiales, las cuales se encuentran explícitas en la misión de la escuela de medicina de la Universidad Juárez Autónoma de Tabasco (UJAT, 2020), misma que comparte con otras instituciones: “1) Formar médicos generales que contribuyan de manera significativa en la prevención y atención de los problemas de salud del individuo y de la sociedad. 2) A través de una formación sólida e integral, capaces de aprender de manera permanente y autónoma. 3) Con alto sentido humanístico, científico, tecnológico y ético". Esta propuesta parte de un significado de la salud en sentido negativo, es decir, de la presencia de la enfermedad.

Aunque es reconfortante analizar estos enfoques, quedan descontextualizados del concepto de salud positivo de la Organización Mundial de la Salud (OMS): "es un estado de completo bienestar físico, mental y social, y no solamente la ausencia de afecciones 0 enfermedades" (OMS, 2020a), ya que la salud debe ser el objeto de estudio $y$ el objetivo de quien aprende, no en el sentido de determinar la normalidad, sino de transformar su práctica en función de la comprensión de los determinantes sociales de la salud:

Son las circunstancias en que las personas nacen, crecen, viven, trabajan $\mathrm{y}$ envejecen, incluido el sistema de salud. Esas circunstancias son el resultado de la distribución del dinero, el poder y los recursos a nivel mundial, nacional y local, que depende a su vez de las políticas 
adoptadas. Los determinantes sociales de la salud explican la mayor parte de las inequidades sanitarias, esto es, de las diferencias injustas y evitables observadas en y entre los países en lo que respecta a la situación sanitaria. (OMS, 2020b).

Estos problemas sociales producen una gran desventaja de la mayor parte de la población mexicana ante la pandemia de COVID-19. La inflamación sistémica de bajo grado con elevación de marcadores como interleucina 6 , proteína C reactiva y factor de necrosis tumoral alfa, condiciona la aparición del "síndrome de citocinas" (Amezcua, 2020). Este fenómeno fisiopatológico explica la entrada a mayores niveles de gravedad de los pacientes infectados.

\section{| Material y métodos}

Se hizo un estudio cualitativo, descriptivo, desde la hermenéutica (Monje, 2011, p. 11), donde se analiza la construcción del modelo de educación médica de Flexner que data de principios del siglo XX (Flexner, 1910), así como de los determinantes socialmente construidos que lo hacen persistir. El análisis se hizo desde tres enfoques para la aplicación de los recursos tecnológicos: el trabajo en el aula, el currículo y la educación en valores. El desempeño de la escuela de medicina se analizó desde el modelo del estructuralismo funcional de Talcott Parsons (1902-1979), acerca de las necesidades sistémicas o mantenimiento de pautas y control de tensiones, que se expresa mediante el acrónimo AGIL por las siglas en inglés de Adaptation, Goal Attainment, Integration, Latency (Girota, 2010).

En este contexto, los ámbitos en que se realiza este análisis, a partir de los propósitos de la Misión de la escuela de medicina, son: 1) El uso de los recursos para la transición a ambientes de aprendizaje donde el docente acompañe a los estudiantes en los procesos de identificar, seleccionar, y hacer uso crítico de la información; 2) La reconstrucción de un currículo de emergencia donde las asignaturas se adapten a la realidad y se implementen la educación a distancia, impartiendo capacitación docente en el uso de las TIC y la formación pedagógica para aplicar las temáticas con 
problemas situados, construidos con información de la realidad y que puedan ser analizados desde diversos niveles de aproximación; y 3) La aplicación de estos problemas contextualizados descriptivamente y consensuados por el equipo docente, lo que permitirá el análisis de las implicaciones desde los aspectos básicos científicos a los campos de la ética y la bioética que incluyen el análisis explícito de los valores humanísticos, los derechos y obligaciones del ser humano universal con su medio ambiente.

\section{Fundamento de la educación médica tradicional}

Los datos científicos muestran que las actividades de memorización acrítica no producen aprendizaje. Mientras que las prácticas tradicionales parten de la idea de que el conocimiento se puede transmitir por el docente y lo adquiere el estudiante, los hallazgos de las neurociencias muestran la complejidad de las estructuras que se movilizan para el aprendizaje pero que requieren de un hacer para conformar el aprendizaje duradero (Bransford \& Brown, 2000). La manera tradicional de que la educación médica sea científica es con la inserción de cursos fragmentados de ciencia y no mediante el aprendizaje de la aplicación de la ciencia en la clínica. Por ello fracasa en sus intenciones e incluso esos conceptos, memorizados de manera enciclopédica, no adquieren sentido (Granados, 2009) y se describen como intrascendentes para la práctica (Watmough et al., 2010).

La educación médica dominante se llama tradicional, pues sigue aún en el siglo XXI la corriente filosófica del positivismo de Auguste Comte (17981857) que permeó en la construcción del modelo de Abraham Flexner (1866-1959), que sigue presente 110 años después de su puesta en marcha (Flexner, 1910). Para esta corriente de pensamiento, que además sustenta el conductismo, se promueve el estudio de la ciencia con el abandono del análisis de las situaciones sociales en su complejidad. Si bien el positivismo tuvo una contribución en la aplicación del método científico de la experimentación, este tipo de estudio que intenta sistematizar el conocimiento carece de la suficiente capacidad de examinar la realidad desde una perspectiva compleja. 
Esta filosofía fue fundamento para la construcción de utopías tecnocráticas y fue básica para la conformación de la Modernidad, pues su lema fue orden y progreso, mismo que aún se conserva en la bandera de Brasil. La sola extrapolación de los métodos de las ciencias de la naturaleza, donde la biología era la de más reciente aparición, no permite examinar a la persona que padece, que es objeto de estudio de la medicina. El modelo Flexner estaba orientado a establecer orden en la multitud de programas educativos presentes a finales del siglo XIX, y provocó el cierre de muchas escuelas de medicina, así como progreso en el sentido de insertar conceptos de las llamadas ciencias de la naturaleza en la práctica de la medicina.

La mayor parte de las instituciones de educación médica del mundo se encuentran conformadas en dos bloques emanados de las observaciones del Informe Flexner (Irby et al., 2010). Orientado desde las conceptualizaciones del positivismo, que ese tiempo fueron sistematizadas por John B. Watson (1878-1958), donde lo principal es observar una conducta a expensas de la puesta en presencia de un concepto, disciplina o sistema de referencias. Este cambio de conducta se espera que ocurra de manera automática una vez que se muestra el estímulo, sin que sean importantes las modificaciones cognitivas al interior de los aprendices. Esto se denomina acondicionamiento y se espera como repuesta de los estudiantes porque van a ponderar el conocimiento que se les presenta.

La imposición de estas doctrinas determinó el currículo de la medicina que se ha mantenido durante más de cien años en muchas escuelas. Consta de dos bloques: el básico, en el cual se enseñan conceptos que se espera que los estudiantes internalicen para comprender el siguiente bloque de conceptos; el segundo bloque es el clínico, en el cual se espera que los estudiantes apliquen lo que memorizaron años antes. Esta disposición que estructura el currículo, no solo de medicina sino de la mayor parte de las profesiones, parte de la idea ingenua de que si se hace memorizar conceptos de la ciencia (en forma de definiciones, esquemas y otros) antes que los contenidos conceptuales y procedimentales de la profesión, 
se piensa que los estudiantes los aplicarán una vez que se encuentren ya en el tercer año de su trayectoria escolar, en las cuestiones que, en el bloque clínico deberán adoptar de manera significativa a su vez (en forma de definiciones, listado de signos y síntomas, entre otros). Esto no sucede de esa manera idealizada. En las escuelas tradicionales son comunes las quejas de los docentes de cursos superiores acerca de la velocidad del olvido de los contenidos de los primeros cursos (Rodríguez, 2014; Torres et al., 2011).

\section{Los recursos tecnológicos y la reorientación de las actividades en el aula}

El primer propósito escrito en la Misión de la escuela de medicina (UJAT, 2020) es "Formar médicos generales que contribuyan de manera significativa en la prevención y atención de los problemas de salud del individuo y de la sociedad", cuya pertinencia y eficiencia se encuentran normadas por la Asociación Médica Mundial (AMM, 2020a). Esto parece no corresponder a la realidad epidemiológica del país. Los esfuerzos formativos al parecer no se dirigen a atender los grandes problemas nacionales, que en el caso de las ENT son pandemia. La observación de datos referentes a las ENT muestra que éstas no solo no disminuyen, sino que la proporción de población afectada aumenta. En lo referente a la emergencia causada por la pandemia de COVID-19 en 2020, hubo necesidad de establecer todas las clases en un sistema en línea de manera apresurada. Esto presentó sus propios retos y amenazas. Uno de los problemas primordiales fue que las clases en línea fueron difíciles de seguir por $67 \%$ de los estudiantes en la principal institución educativa a nivel superior de México, la Universidad Nacional Autónoma de México (UNAM) (Román, 2020a). Esto sucedió porque muchos de ellos no disponían de dispositivos móviles y sus teléfonos inteligentes carecían de acceso a la red de Internet por falta de recursos para adquirir datos. Por otra parte, se encontró que muchos docentes no podían apoyarlos porque carecen de habilidades e interés en el uso de los recursos en línea (Román, 2020b).

En el modo tradicional de las clases en el aula, presenciales, se tienen tres aspectos fundamentales. Para Díaz-Barriga la escuela ha dejado 
en el olvido que su función es formar y educar. En este caso cumplir con la función de promover el aprendizaje de las cuestiones relacionadas a la salud y la enfermedad. Pero la escuela se centra en "rituales tradicionales de la escuela anquilosada” (DíazBarriga, 2020, p. 25). Estos rituales son el cumplimiento estricto de los horarios, los actos para el ingreso al aula, la toma de apuntes, el cumplimiento de las tareas, así como en la presentación de exámenes parciales y finales. En la escuela tradicional no se desarrollan los procesos educativos en función del aprendizaje situado. En estas instituciones, la actividad "arquetípica es muy diferente de una actividad auténtica”, pues aquella realiza actividades "distintas de las que los practicantes auténticos realizan” (Sagástegui, 2004, p. 31).

Estas formas de desarrollar las clases se sustentaban en el conductismo, donde la función del profesor se enfocaba a impartir la cátedra y solo presentaba los materiales para que los estudiantes los aprendieran. Sin embargo, estas formas del conductismo se perdieron en la entrada a la posmodernidad. Ahora, la forma de clases presenciales es donde el docente solo es la autoridad que mantiene el control y el orden, pero cuya actividad de dictado de la cátedra se disuelve ante la exposición de los temas por parte de los estudiantes. Esto lo hacen los docentes con la excusa de que así los hacen responsables de su aprendizaje. El resultado es que se maneja información fragmentada, sin el desarrollo de "capacidades para anticipar, formular e incidir sobre problemas sociales cada vez más desiguales, lábiles e inciertos” (Sagástegui, 2004, p. 31). Esta no es una práctica infrecuente donde la única aplicación de los recursos tecnológicos consiste en encontrar textos y figuras para las diapositivas y usar un proyector con auxilio de una computadora portátil. Por tanto, los estudiantes no adquieren sentido al interior de la cultura en la cual se desarrollan en la vida real (Seely et al., 1989). Estos "vicios de la educación”, como es el manejo enciclopédico de los contenidos (Díaz-Barriga, 2011, p. 15), plantean retos para aplicar estrategias didácticas híbridas para un tránsito productivo de presencialidad a virtualidad (Chehaibar, 2020, p. 90). 
Para una práctica que produzca una mejor aplicación de los conceptos de las ciencias en el campo de la medicina, se debe partir de preguntas relacionadas a lo que se quiere que los estudiantes aprendan. Se sugiere plantear situaciones que se han de resolver con acuerdo a las necesidades de la sociedad, principal agente ante la cual los procesos educativos son responsables, especialmente la universidad pública. El aprendizaje situado exige una interpretación del mundo semejante a las formas de aprendizaje que se generan en la vida cotidiana y en el marco de una cultura donde se interactúa con agentes humanos y no humanos (Sagástegui, 2004, p. 35). Con el consenso del equipo de docentes se han de plantear las siguientes cuestiones con diferentes niveles de profundidad, según la experiencia y trayectoria que tienen los estudiantes:

1) ¿Cómo es la realidad inmediata (como enfermedades o factores de riesgo) sobre la que se quiere intervenir? 2) ¿Qué factores contextuales la condicionan? 3) ¿Cuál es el juicio o evaluación que se hace de la situación problema (que no necesariamente es sencilla o unívoca)? 4) ¿Cuáles son los recursos y medios operacionales (información) que se disponen para actuar en función de la resolución de los problemas y/o la satisfacción de las necesidades o carencias detectadas (de los pacientes)? 5) ¿Cuáles son los factores más relevantes, que probablemente, han de actuar de manera positiva, negativa o neutra, con relación a los objetivos o finalidades propuestas dentro del plan de manejo a realizar para resolver los problemas de los pacientes? (Muñoz et al., 2020, p. 176).

No se debe evitar el uso de los recursos tecnológicos en el aula sino lo contrario, aplicarlas en el aula. Hay que convertirlas en herramientas de aprendizaje, por lo que se debe trabajar intelectualmente con ellas para el desarrollo de capacidades que permita la aplicación de la información en problemas de salud (Muñoz \& Maldonado, 2011; 2017; Muñoz, 2012; Muñoz et al., 2012), que los estudiantes deben aprender a percibir como tales (Muñoz et al., 2014). Y ya que no se puede 
tener dominio de todas ellas y menos de los contenidos en línea, hay que aprender a trabajar con los estudiantes en el sentido de aprender con ellos, de ellos y ellos de los docentes (Díaz-Barriga, 2019, minutos 15 al 19). El uso de los recursos tecnológicos no ha de ser para sustituir a los docentes ni convertir a las clases presenciales en actividades totalmente en línea donde no se tienen vínculos con el profesor ni los compañeros de clases. Si bien los cursos masivos en línea y abiertos, MOOC por las siglas en inglés de Massive Online Open Courses, así como el desarrollo de actividades en instituciones que ya tenían su preponderancia en línea son funcionales, las instituciones educativas de nivel superior pueden requerir de actividades como en laboratorios o consultorios para el caso de medicina. De esta manera operan desde hace años la Universidad Abierta y a Distancia de México, la Universidad a Distancia de Madrid, la Universitat Oberta de Catalunya, The Open University of London, entre muchas otras.

La estrategia para las actividades escolares se puede aplicar mediante la lectura de artículos científicos, videos seleccionados, blogs elaborados por divulgadores reconocidos, páginas web de sociedades 0 instituciones de prestigio. Las actividades en clase, o mediante los dispositivos tecnológicos en su caso, se refieren a la elaboración de las tareas en el aula, ya que tendrán información relevante, aunque puede no ser suficiente. Esto supera los problemas de copiado y pegado, que son atribuibles al docente y no a los estudiantes (Díaz-Barriga, 2019, minutos 21 a 24). Los estudiantes expresan que las causas del copiado o pegado se deben a la certeza de que el docente no va a leer la tarea, no se entiende lo que el docente trata de explicar en clase, tienen demasiadas tareas de tantas asignaturas y deben entregar "algo". Por ello los docentes deben planear actividades donde los estudiantes puedan reflexionar acerca de la información útil para aplicar en una situación específica de la vida real, donde lleguen al aula o al espacio virtual de clases ante el profesor, a trabajar y no a escuchar disertaciones pues esa es una práctica del siglo XVII (Díaz-Barriga, 2019, minutos 21 a 24).

A esta clase de diseño se le llama flipped classroom o aula invertida (Espinosa et al., 2018; Moya \& Williams, 2016) con apoyo de una plataforma en línea. En las clases 
invertidas el papel del profesor en la sala es el de auxiliar a los estudiantes y no el de transmitir información (Bergmann \& Sams, 2012). Ya sea en este tipo de clases o en otras de tipo dinámico, donde se puede realmente hacer que los estudiantes cumplan con el compromiso que ellos sean responsables de su formación y educación. Esto también tiene el sentido de proporcionarles las herramientas necesarias para que puedan seleccionar, jerarquizar, discriminar y aplicar información a problemas de la vida real. Se puede poner en marcha en una secuencia didáctica con los siguientes elementos: 0) Ubicación curricular, 1) Suscitador significativo, 2) Dilema, 3) Investigación dirigida, 4) Clase colaborativa, 5) Metáfora didáctica o contextualización, 6) documentación complementaria, 7) Puesta a prueba, 8) Referenciación (Figura 1).

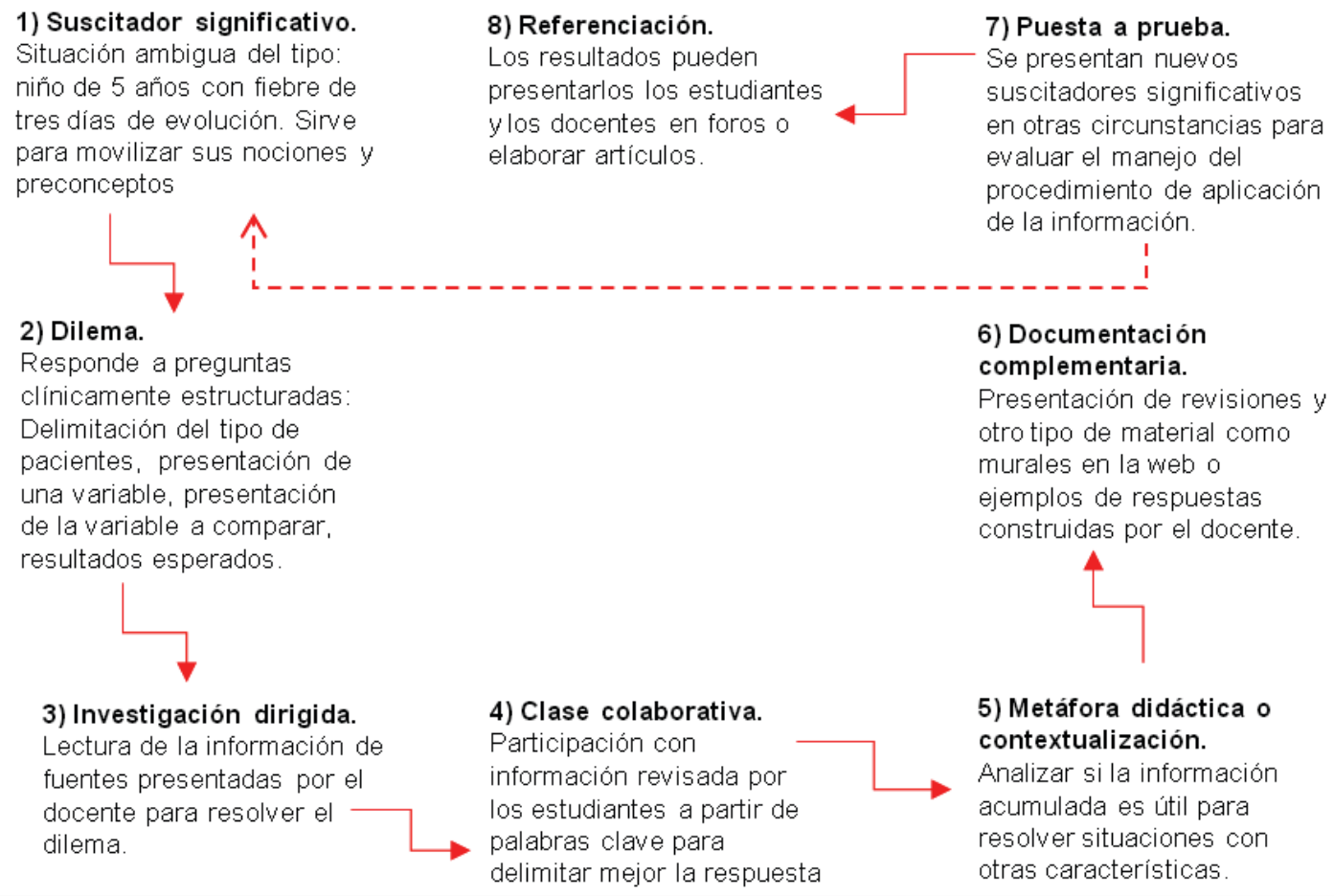

Figura 1. Modelo de secuencia didáctica no lineal.

Otra estrategia dinámica es el el eje del ABP es un problema o aprendizaje basado en problemas situación problema que tiene más (ABP). Habitualmente se conforman de una solución la cual sirve como grupos de 8 a 10 estudiantes y motivo para reflexionar y encontrar 
respuestas por lo que tiene un enfoque probabilístico que se sustenta en la búsqueda de datos con rigor científico para sus soluciones. Este enfoque tiene pasos sistematizados y parte de situaciones problemáticas ambiguas, ya que deben discutirse las diversas opciones con base en datos con rigor científico, del tipo de: “mujer joven con dolor agudo en fosa ilíaca derecha y sangrado genital” (Mendoza et al., 2015, p. 236) (Tabla 1).

Tabla 1

Usos de los recursos tecnológicos en el desarrollo pedagógico

Tipos de enfoque

Problema para resolver

Educación tradicional

Escuela flexible

\begin{tabular}{|c|c|c|}
\hline $\begin{array}{l}\text { Construcción de } \\
\text { conocimiento a partir de } \\
\text { diversas fuentes donde los } \\
\text { límites de las asignaturas } \\
\text { se borran; estrategias de } \\
\text { búsqueda en literatura } \\
\text { científica para jerarquizar, } \\
\text { discriminar y seleccionar }\end{array}$ & $\begin{array}{l}\text { Exposición de información } \\
\text { fragmentada, multimedia y } \\
\text { presencial de una asignatura, } \\
\text { donde el profesor "dicta su } \\
\text { material instruccional” en línea } \\
\text { como en Vargas et al. (2020). }\end{array}$ & $\begin{array}{l}\text { Resolución de problemas } \\
\text { mediante la búsqueda en } \\
\text { fuentes de información } \\
\text { y de disciplinas distintas } \\
\text { (Muñoz \& Maldonado, 2011), } \\
\text { resolución de preguntas } \\
\text { clínicamente estructuradas } \\
\text { (Muñoz et al., 2020). }\end{array}$ \\
\hline
\end{tabular}

la información pertinente.

Formación de capacidades tanto profesionales como transversales con base en los enfoques constructivistas.
Se promueve la adquisición de información más que la de capacidades del aprendizaje autónomo, o las necesarias para el desempeño profesional, como en Vergara y Carrillo (2018).

En los exámenes y la de tareas en línea, a semejanza de cursos presenciales, se encontró "copia flagrante y evidente de contenidos" (Vargas et al., 2020), lo cual es atribuible a los docentes por fallas en el diseño de las experiencias (DíazBarriga, 2019, minutos 21 a 24).
Aplicación de información recuperable en línea en situaciones problemáticas similares a las de la vida real, tanto ambiguas (Mendoza et al., 2015), como situadas (Muñoz, 2012).

Discusión diacrónica de situaciones desde distintas perspectivas, donde analizar valores para construir una posición ética, como de interculturalidad (Muñoz et al., 2014), o de consumo de alimentos respecto a calentamiento global. 
2. Los recursos tecnológicos y la reconfiguración del currículo

El segundo propósito de la Misión es: educar médicos "A través de una formación sólida e integral, capaces de aprender de manera permanente y autónoma”. El currículo actual de la educación médica se basa en una interpretación de las recomendaciones en el Informe de Flexner, pero desde una perspectiva anterior a la modernidad. Si se revisan con atención esas recomendaciones, se encuentra que afirma la necesidad de insertar los avances de la ciencia en las actividades habituales de los médicos. Si bien, de manera explícita el informe propone dos años de ciencias básicas y dos años de clínicas, también dice que ambos tipos de conocimiento deben estar integrados a lo largo del currículo de cuatro años, así como otras características que corresponden más a un pensamiento constructivista. Aunque Flexner no tuvo contacto con las ideas de estas corrientes en la época de la publicación del informe, pues Jean Piaget (1896-1980) publicó acerca de sus ideas del desarrollo de los niños hasta 1920, y Lev Vigotsky (1896-
1934) permaneció sin conocerse en occidente durante muchos años, pues sus aportaciones acerca de que se aprende en función de los otros se difundieron hasta 1960, sus ideas de un aprendizaje activo permanecen sin atenderse.

Por otra parte, al analizar la estructura curricular, se encuentra, para la época de la publicación del informe, persistencia del inductivismo, previo a la epistemología de Claude Bernard (1813-1878), que propone la falsación de las hipótesis 70 años antes que Carl Popper (19021994). Bernard delimitó las condiciones de desarrollo de la fisiología experimental, pues trazó un camino para un dominio de investigaciones que no puede limitarse a un estudio fisicoquímico de los seres vivos; varias de las recomendaciones de Flexner no se introdujeron en el currículo. De esa manera la anatomía, centrada en la descripción de las estructuras, se mantuvo como una ciencia básica, dado que quienes participaron en la construcción del currículo no tomaron en consideración que las disciplinas morfológicas "son leyes durmientes 
o expectantes que no impiden ni producen ningún fenómeno" (Caponi, 2001, p. 399). Esa idea acerca de las estructuras morfológicas en realidad corresponde a una epistemología anterior, cuando desde el inductivismo se afirmaba que dado un análisis anatómico era posible describir una función. Esta idea, que fue superada por Bernard mediante la promoción de la fisiología experimental como ciencia que explica cómo los fenómenos ocurren y la distingue de cualquier especulación metafísica sobre cómo ocurren (Caponi, 2001), sigue viva y saludable hasta esta época.

Las características de las recomendaciones del informe que no se han atendido en la mayor parte de las escuelas de medicina continúan siendo innovadoras:

Debe alcanzarse la integración de las ciencias básicas y las ciencias clínicas en los cuatro años. Debe estimularse el aprendizaje activo. Debe limitarse el aprendizaje de memoria mediante conferencias. Los estudiantes no deben aprender solamente hechos, sino desarrollar el pensamiento crítico y la habilidad de resolver problemas. Los educadores deben enfatizar que, en los médicos, el aprendizaje es una tarea para toda la vida. (Vicedo, 2002, p. 158).

Estas recomendaciones se contraponen a las características de las estructuras de las universidades, que, siguiendo la teoría de los sistemas sociales, se analizan mediante el modelo AGIL de Parsons:

1) Adaptación. Las administraciones escolares adaptan las situaciones de la vida real y los problemas de salud de la sociedad a la estructura del currículo escolar, de esta manera se han dejado de observar los problemas primordiales de salud de los mexicanos: las pandemias de ENT (INSP, 2020; Rosas \& Borrayo, 2018) y la crisis de pobreza y pobreza extrema (CONEVAL, 2020), además de los efectos del cambio climático que se producen también por hábitos de consumo de alimentos exfoliadores del ambiente que caracterizan al Antropoceno (Willet et al., 2019). 
2) Metas y logros. Los estudiantes trabajan mediante la expectativa de la obtención de su titulación y no porque se preocupen por su formación o educación. Los estudiantes se preocupan por lo que van a responder en los exámenes que dominan la actividad escolar y menos por comprender el contexto en que viven y las explicaciones con base en datos de esa realidad en la que viven y que los rebasa. No por otra parte, hasta $30 \%$ de los trabajadores de la salud fueron confinados en sus casas por la presencia de factores de riesgo, no solo por la edad (IMSS, 2020).

3) Integración. Más que promover ambientes donde los estudiantes y los docentes transiten a ambientes de mayor desarrollo cognitivo, en la universidad se mantienen los roles de discriminación, xenofobia, sexismo, verticalidad en las relaciones entre estudiantes y docentes, pues los principales modos de dominación social son el neoliberalismo, el colonialismo y el patriarcado (De Souza,
2020, p. 35). Estos fenómenos se refuerzan mediante la penalización a la disidencia, desde la práctica del bullying y las "novatadas" para los estudiantes, y la marginación y bloqueo académico a los profesores. Por supuesto que estas prácticas son invisibles. Esta integración la explica Emile Durkhein (1858-1917) en función de la educación no como proceso formativo o educativo. Como en la novela "1984", de George Orwell (19031950), la educación desde este enfoque de análisis es un proceso de socialización diferencial desde la división del trabajo y consecuentemente de los niveles de la sociedad que deriva en jerarquización y desigualdad, por lo que debe haber escasa movilidad social (Usagui, 2005), pues de otra manera se corre el riesgo del desorden, como afirma Parsons en su modelo.

Para las instituciones de educación, esas diferencias se califican desde el criterio de calidad que evalúa las deficiencias que evidentemente 
hay en las regiones desfavorecidas de México. Este uso de las evaluaciones de calidad hace calificar "como justas estas diferencias que corresponden a un arbitrario cultural y otros arbitrarios culturales” (Plá, 2018, p. 27). Lo que avala la calidad no son entonces los logros cognitivos 0 académicos de los egresados o de los profesores de las instituciones estigmatizadas como de baja calidad, sino las deficiencias respecto a un referente, la supra cognición, que es "una dimensión política mediante la cual se define un sujeto cognitivo universal y por lo tanto se ubica por encima de las otras normas culturales de cognición” (Plá, 2018, p. 107).

4) Latencia. Pues los intentos de flexibilidad, cambios en la estructura curricular no permean, sino que se retoman las conductas habituales que hacen que se mantenga el currículo petrificado de las disciplinas en estructura secuencial, que ha demostrado su falta de efectividad. Por ello en la mayor parte de las escuelas de medicina se mantiene el esquema lineal a pesar de que se han puesto en marcha proyectos con el currículo espiral y el aprendizaje con base en problemas, como en las universidades de McMaster en Canadá, de Limburgo en Maastricht en Holanda y en el Tecnológico de Monterrey en México. Este enfoque inició en 1969, "ante la necesidad de reorientar la educación médica para formar médicos integrales y resolutivos" (Mendoza et al, 2012, p. 235). Estas innovaciones continúan invisibles para muchas de nuestras escuelas de medicina.

Construir un currículo que atienda estas recomendaciones en la educación médica requiere de una visión integradora. En este, el enfoque ha de ser interdisciplinar, a fin de cumplir con los propósitos del perfil de egreso (Figura 2). Una opción es la estructura espiral, donde, como recomendó Juan Amós Comenio (1592-1670), debe enseñarse primero lo simple y transitar a lo más difícil. Un currículo espiral, a la manera de una doble hélice de DNA, mantiene la unión 
entre las moléculas precedentes con las consecuentes por medio de enlaces, en este caso, pedagógicos y conceptuales. En las primeras etapas los problemas que enfrentan los estudiantes son simples, en las intermedias, complicados, y en los finales, complejos (Muñoz, 2012). Por ello se deben:

Incorporar retos transversales y dinámicos en los planes $\mathrm{y}$ programas de estudio, equidad de género, derechos humanos, democracia, sustentabilidad, aplicación de avances científicos y tecnológicos; reformar el andamiaje de las universidades para dar sustento real a la flexibilidad, que supere el formato de contenidos mínimos, número de créditos y formas de acreditación/certificación que promuevan la interdisciplina (Chehaibar, 2020, p. 90).

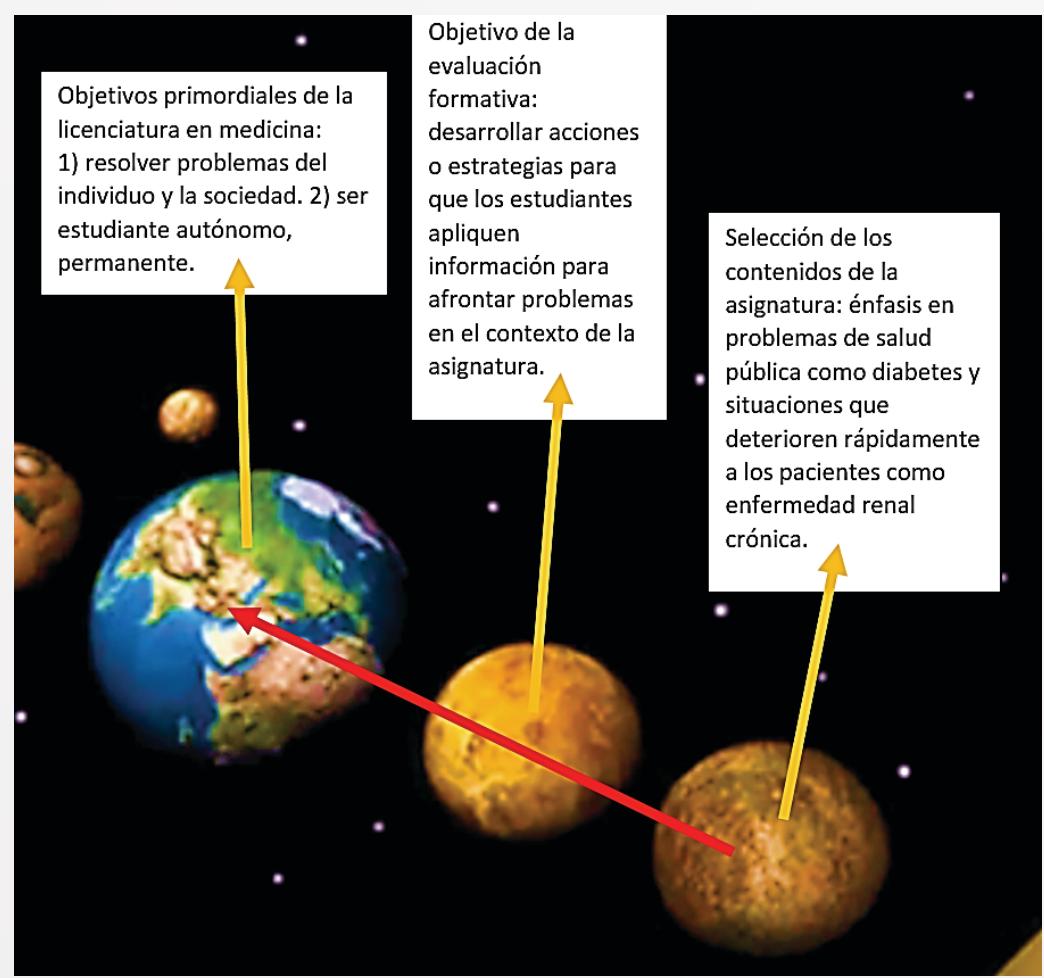

Figura 2. Alineación del currículo y la evaluación con los objetivos de la educación médica.

Trabajar en situaciones donde se fomente la interdisciplina puede realizarse mediante el aprendizaje en el contexto de la práctica de una Medicina con Base en Datos, Hechos o Evidencia Científica (Evidence-Based 
Management) (Kepes et al., 2016). Esta se desarrolla desde una práctica de la medicina sustentada en la Educación Médica Basada en Evidencias de alto rigor científico (Best Evidence Medical Education) (Sánchez, 2012). A pesar de las críticas al positivismo lógico o neopositivismo, a su vez fuente teórica del pensamiento fundamentalista, que fue la corriente cultivada por Bertrand Russell y los miembros de la escuela de Francfort, este enfoque es un avance respeto al paradigma del determinismo, sustentado por la presencia de las bacterias que causan enfermedades, pues es un enfoque probabilístico donde confluyen factores de riesgo y redes causales (Ortega \& Cayoela, 2002), de mayor valor para elaborar y corroborar hipótesis clínicas acerca de los pacientes. Una de las aportaciones de Popper es que la ciencia no ofrece un conocimiento definitivo, sino que se encuentra direccionado a una acción crítica y persistente que obliga a mantener una teoría en modo abierto a su posible falsación (Ortega \& Cayoela, 2002).

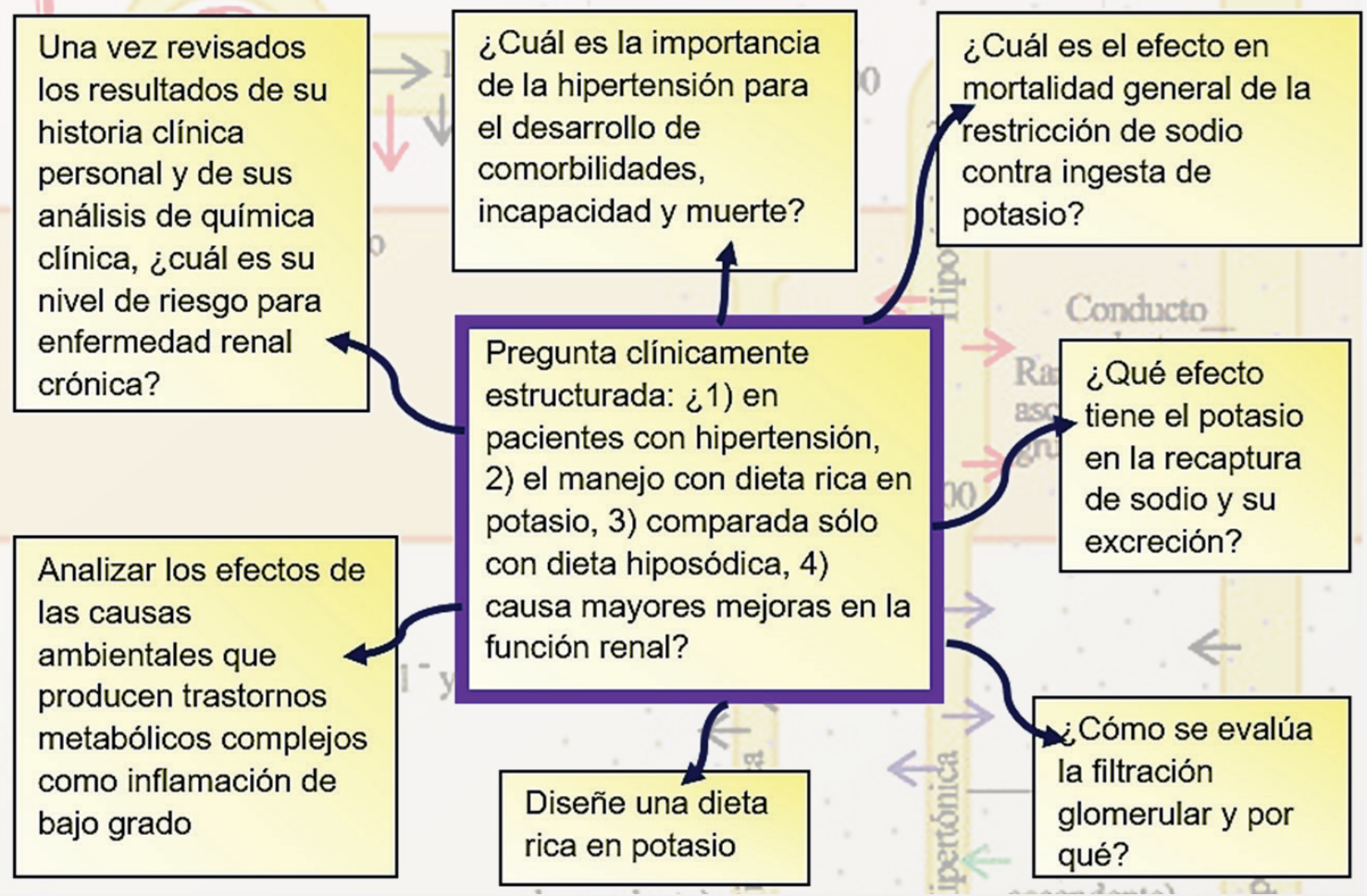

Figura 3. Aplicaciones de una pregunta clínicamente estructurada. 
Una de las aplicaciones del enfoque de la Educación Médica Basada en Evidencia, es el planteamiento de preguntas clínicamente estructuradas. Las preguntas constan de cuatro partes. La primera se define en términos de una población que se limita en términos de condiciones de edad, factores de riesgo y condiciones de salud. Por ejemplo, pacientes con enfermedad renal crónica. La segunda parte introduce la intervención contra el manejo habitual, la exposición a un patógeno, a condiciones ambientales, entre otras: el uso de la pentoxifilina. La tercera plantea el reto: comparada contra el manejo de dieta y normotensores. La cuarta parte es un dilema: va a mejorar (o no) los indicadores de la función renal (Sánchez, 2012). Tal como se observa en la figura 3, una pregunta puede analizarse desde diversos niveles y enfoques. De esa manera es posible revisar cuestiones de fisiología y de salud pública a partir de una pregunta inicial con el auxilio de la información en la red.

Para aumentar la eficiencia el docente debe convertirse en el experto en la búsqueda de información científica en la red, en un acompañante de los estudiantes en la calificación de la información con base en el rigor científico con que fueron construidos los datos. La experticia de los estudiantes es el dominio de los recursos tecnológicos, pero no de la identificación de la información necesaria, depende el que ellos aprendan un proceso de búsqueda cada vez más complejo donde se les hace transitar hacia la autonomía (Tabla 2). De esa manera, al elevar los estándares de la información, es posible gestionar, aplicar y difundir conocimientos altamente relevantes (Kepes et al., 2016).

Tabla 2

Tránsito de los datos a la sabiduría

\begin{tabular}{llrllll}
\hline \multicolumn{1}{c}{ Datos } & \multicolumn{2}{c}{ Información } & \multicolumn{2}{c}{ Conocimiento } & \multicolumn{2}{c}{ Sabiduría } \\
\hline Cifras sin procesar & Significación de & Uso & de & la & Uso del conocimiento \\
o interpretar, sin & los datos para una & información para & para mejorar la calidad de \\
juicios de valor & necesidad específica & tomar decisiones y & vida humana ylanetaria \\
& & \multicolumn{3}{c}{ acciones } \\
\hline \multicolumn{2}{c}{ Consumidores de información } & \multicolumn{3}{c}{ Prosumidores de conocimiento } \\
\hline
\end{tabular}


Este tipo de trabajo escolar se aproxima a la construcción de las competencias transversales de la educación médica: 1) resolver problemas clínicos habituales y derivar correctamente los de las especialidades, 2) aprender cómo acceder al conocimiento y acumularlo en forma de una red semántica y de una consciencia cognitiva, 3) aprender el cuidado de los pacientes y atenderlos desde posiciones basadas en la bioética, 4) trabajar en equipos de inter y transdisciplina, 5) manejo de los recursos tecnológicos para acceder y difundir el conocimiento, 6) aproximaciones a la investigación clínica, sobre todo para sustentar el quehacer del médico en la identificación de datos con rigor científico (Kebede \& Pronovost, 2015; Pershing \& Fuchs, 2013).

\section{Los recursos tecnológicos y el análisis de los valores de la medicina}

Los recursos tecnológicos tienen aplicación también para cumplir con el tercer propósito de la Misión de la escuela de medicina (UJAT, 2020): formar médicos "Con alto sentido humanístico, científico, tecnológico y ético”. Una primera aplicación es discutir los discursos de los filósofos y teóricos de las ciencias sociales acerca de la medicina. En este aspecto es necesario discutir los postulados del Juramento, erróneamente atribuido a Hipócrates en muchas escuelas de medicina, en realidad construcción de la Asociación Médica Mundial en 1948, y cuya última revisión es de 2017, en la ciudad de Chicago, Estados Unidos (AMM, 2020b).

Los recursos tecnológicos y el acceso a la red permiten la construcción de un pensamiento diacrónico (Díaz-Barriga, 2019, minuto 25). Una pregunta, sobre todo en el campo de la ética médica y de la bioética, es posible responderla de más de una manera. Esto significa que posibilita la construcción de respuestas con base en diversos enfoques epistemológicos, sin que se deba descalificar lo que no se consideraba la respuesta unívoca. Porque desde el manejo tradicional, lo que se fomenta desde los exámenes departamentales, las preguntas contenidas en bancos donde se trata de que se responda muchas veces de la manera en que piensa el profesor, más que desde las interpretaciones de los 


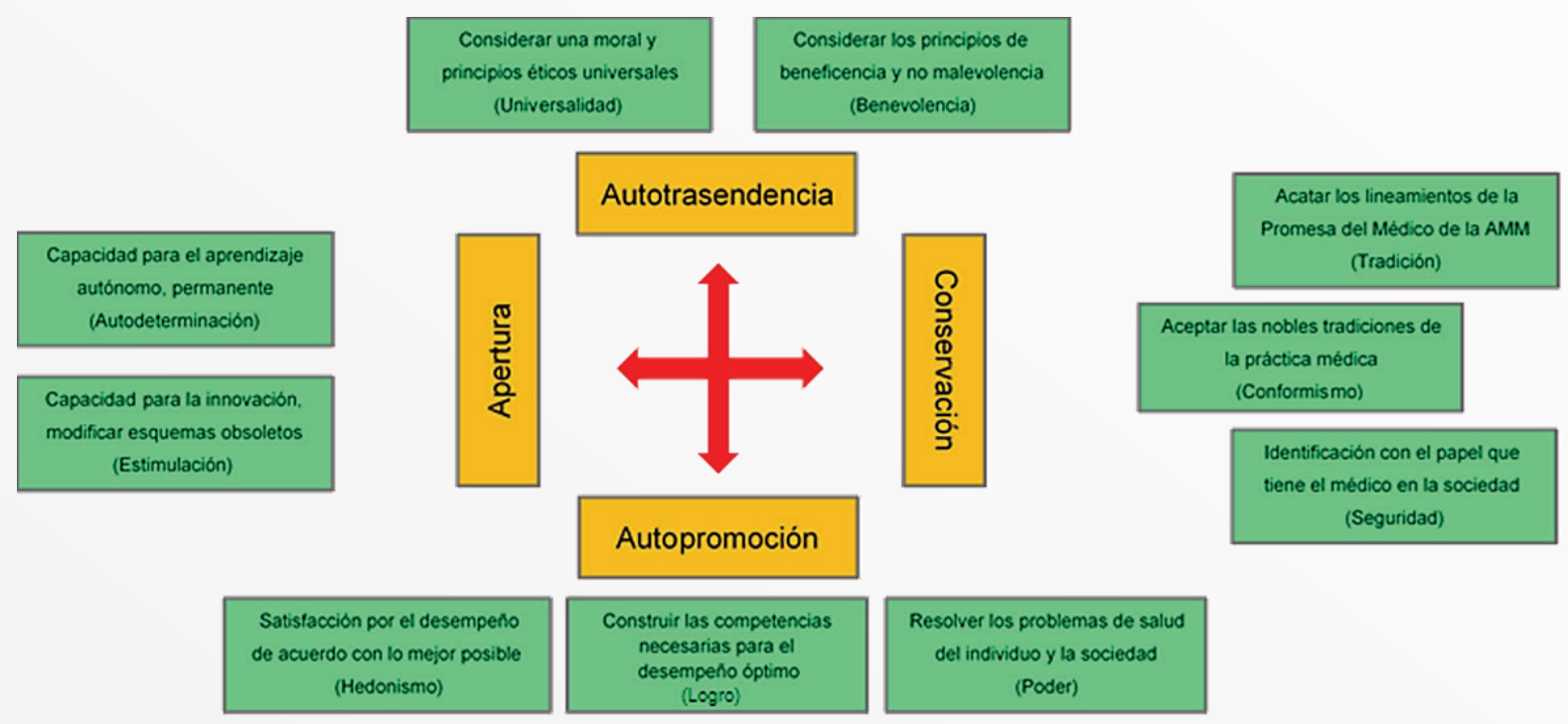

Figura 4. Esquema de valores en medicina, adaptado de García (2005).

científicos y los filósofos, es un pensamiento sincrónico, totalmente acrítico, donde los valores que se promueven son principalmente la competencia y el darwinismo social (Vargas, 2005) (Figura 4).

En este aspecto son muchos los discursos que ponderan las actividades del médico desde una perspectiva integrada, por ejemplo, William Osler (1849-1919) en 1903:

Se ha hecho mucho, pero mucho queda por hacer; un camino ha sido abierto, y las posibilidades para el desarrollo científico de la medicina parece que no tuvieran límite. Excepto en su aplicación, como médicos generales, no tendréis mucho que ver con esto. Vuestro es un deber más alto y sagrado. No penséis en encender una luz que brille ante los hombres para que puedan ver vuestras buenas obras; al contrario, pertenecéis al gran ejército de trabajadores callados, médicos y sacerdotes, monjas y enfermeras, esparcidos por el mundo, cuyos miembros no disputan ni gritan, ni se oyen sus voces en las calles, sino que ejercen el ministerio del consuelo entre la tristeza, la necesidad y la enfermedad. (Citado en Gutiérrez, 2008, p. S15).

El discurso de Von Weizsaecker (1886-1957) en 1927: 
Aprendimos que el cuerpo humano se compone de tejidos y que los tejidos se componen de sustancias químicas. Aprendimos que todo esto se modifica en las enfermedades con acuerdo a la forma y la composición. Ahora podemos emitir un juicio: esto está enfermo. Pero el enfermo puede decir: yo estoy enfermo. ¿Es que una célula puede decir "yo"? ¿Es que una molécula, un átomo, un electrón pueden decir "yo"? ¿Quién es aquel que dice "yo"? Solo nos enseñaron cuestiones acerca de que las cosas son "algo", no aprendimos nada de cosas que son "alguien”. Pero la consulta comienza con que alguien nos dice "Estoy enfermo", y nos asombramos de que no nos desconcertemos inmediatamente, dado que no hemos aprendido nada de eso; si fuéramos sinceros deberíamos estar desconcertados. (Díaz, 2012, p. 35).

Y la respuesta de Bertrand Russell (1872-1970) a la pregunta de qué mensaje le gustaría que se leyera en el futuro, mencionó la vida cognitiva desde dos ámbitos, la ciencia experimental y las ciencias sociales, y aunque no las articula, si las considera como sistemas paralelos y comunicantes:

Desde el punto de vista intelectual, me gustaría decir lo siguiente: cuando estás estudiando cualquier materia o considerando cualquier filosofía, pregúntate solamente cuáles son los hechos y cuál es la verdad que esos hechos muestran. Nunca te dejes influenciar por lo que tú deseas o por lo que crees, o por lo que crees que traería más beneficios sociales. Toma en cuenta única y solamente los hechos. Eso es el asunto intelectual que tengo que decir.

En cuanto a lo moral lo que tengo que decir es muy sencillo: el amor es sabio y el odio es una tontería. En este mundo en que nos estamos interconectando más y más cercanamente debemos aprender a tolerarnos los unos a los otros, tenemos que aprender a aguantar el hecho de que algunas personas dirán cosas que no nos gustarán. Sólo podemos vivir juntos de esa manera, y si queremos 
vivir juntos y no morir juntos tenemos que aprender esa especie de caridad y tolerancia que es absolutamente vital para la continuación de vida en este planeta. (Russell, 1959, minutos 1 a 2).

Así como la posición del filósofo Emmanuel Lévinas (1906-1996), quien propone como preguntas fundamentales ¿Quién sufre?, ¿qué puedo hacer para disminuir su sufrimiento? "El paciente sufre, se siente degradado, tiene necesidad, pero también está lleno de vergüenza por saber que es un ser necesitado que solicita y demanda ayuda” (Disla et al., 2019, p. 9).

\section{|Conclusiones}

La pandemia de COVID-19 que se dio en México en medio de una pandemia, la de ENT asociadas a la alimentación y la crisis de la pobreza, favorecidas por las prácticas neoliberales, puso en evidencia la necesidad de emplear de una manera más razonada los recursos tecnológicos en la educación superior. Para el caso de la educación médica, es claro que al menos la pandemia de ENT debería estar siendo afrontada de manera que no se tuvieran las cifras tan altas de prevalencia y mortalidad, sin embargo, afecta tanto a los trabajadores de la salud como a la población en general. En este estudio analítico se revisaron las aplicaciones necesarias para el desarrollo de las clases en ambientes escolares más dirigidos a la formación y educación de los estudiantes, especialmente porque se espera que sean estudiantes autónomos, permanentes. Se revisaron las necesidades en la reconfiguración del currículo a una estructura acorde a las posibilidades del siglo XXI, y dejar de lado las tradiciones escolares que datan del siglo XVII que obstaculizan el tránsito a una escuela que verdaderamente construya conocimiento para una práctica de la medicina tendiente al bien común. Esto porque en el análisis desde el modelo del estructuralismo funcionalista, a pesar de no ser totalmente vigente, demostró que la escuela mantiene patrones de comportamiento tendientes a la persistencia de las desigualdades sociales, y que los discursos de calidad propician las inequidades regionales. 


\section{|Referencias}

Amezcua-Guerra, L. M. (2020). Anotaciones breves sobre el síndrome de liberación de citocinas y el bloqueo terapéutico de la interleucina-6 en SARS-CoV-2/COVID-19. Cardiovascular and Metabolic Science, 31(3), 255258. https://doi/10.24875/ACM.M20000067

Asociación Médica Mundial [AMM] (2020a). Declaración de la AMM con normas para el mejoramiento continuo de la calidad de la atención médica. Recuperado de shorturl.at/doCW2

Asociación Médica Mundial [AMM] (2020b). Declaración de Ginebra, enmendada por la $68^{\mathrm{a}}$ Asamblea General de la AMM, Chicago, Estados Unidos, octubre de 2017. https://www.wma.net/es/policies-post/ declaracion-de-ginebra/

Bergmann, J. \& Sams, A. (2012). Flip your classroom: Reach every student in every class every day. Washington: International Society for Technology in Education.

Bransford, J., \& Brown, A. (2000). Cómo aprende la gente: cerebro, mente, experiencia, y escuela. Revista del Instituto de Matemática y Física, 4464. http://matesup.cl/portal/revista/2005/reflexiones.pdf

Caponi, G. (2001). Claude Bernard y los límites de la fisiología experimental. Historia, Ciencia, Saude-Manguinhos, 7(2), 375-406. https://www.scielo.br/ pdf/hcsm/v8n2/a05v08n2.pdf

Chehaibar, L. (2020). Flexibilidad curricular: Tensiones en medio de una pandemia. En Educación y pandemia: Una visión académica (pp. 83-91). ISSUE-UNAM.

Collingwood, R. G. (2004). Idea de la historia. Fondo de Cultura Económica.

De Souza, B. (2020). La cruel pedagogía del virus. CLACSO. http://209.177.156.169/libreria_cm/archivos/La-cruel-pedagogia-del-virus.pdf 
Díaz-Barriga, A. (6 de noviembre de 2019). Reconstruir la docencia: Un reto para el siglo XXI. Segundo Congreso Educación e Inclusión desde el Sur. [Video]. YouTube. Canal oficial del Municipio de Río Grande. https://www. youtube.com/watch?v=kdpcgUlUvTc

Díaz-Barriga, A. (2020). La escuela ausente: La necesidad de replantear su significado. En Educación y pandemia: Una visión académica (pp. 19-29). ISSUE-UNAM.

Díaz-Berenguer, A. R. (2012). ¿Por qué la medicina sigue siendo un arte? Archivos de Medicina Interna, 34(1), 33-35. http://www.scielo.edu.uy/pdf/ ami/v34n1/v34n1a07.pdf

Disla, J., Aranda, C., \& Almánzar, R. (2019). Aportes de la ética levinasiana al cuidado en la terapia contextual. Revista Interdisciplinaria de Filosofía y Psicología, 14(6), 1-14. https://scielo.conicyt.cl/pdf/limite/v14/07181361-limite-14-6.pdf

Espinosa, T., \& Araujo, I. S. (2018). Aula invertida (flipped classroom): innovando las clases de física. Revista de Enseñanza de la Física, 30(2), 59-73. https://revistas.psi.unc.edu.ar/index.php/revistaEF/article/ view/22736

Flexner A. (1910). Medical Education in the United States and Canada: A Report to the Carnegie Foundation for the Advancement of Teaching, Bulletin Number four, 1910. https://www.ncbi.nlm.nih.gov/pmc/articles/ PMC2567554/

García, L. C. (2005). Sistema Valorativo de Estudiantes de Pregrado y Preuniversitarios de la Pontificia Universidad Católica del Perú [Tesis de maestría inédita]. Pontificia Universidad Católica del Perú. Recuperado de shorturl.at/dilvJ

Girota, L. (2010). Talcott Parsons: a propósito de la evolución social. Sociológica, 25(72), 139-165. http://www.scielo.org.mx/scielo. php?script=sci_arttext\&pid=S0187-01732010000100007\&lng=es\&tlng=es 
Granados, J. (2009). La enseñanza de las ciencias básicas en medicina desde la perspectiva de la justificación epistemológica del currículo. Revista Educación, 33(2), 51-60. https://core.ac.uk/download/pdf/204527243.pdf

Gutiérrez, J. A. (2008). La medicina, una ciencia y un arte humanos. Educación Médica, 11(1), 11-15. http://scielo.isciii.es/pdf/edu/v11s1/ mesa1_11s01_ps11.pdf

Instituto Nacional de Salud Pública [INSP]. (2020). Diabetes en México. https:// www.insp.mx/avisos/3652-diabetes-en-mexico.html

Instituto Mexicano del Seguro Social [IMSS]. (2020). Firman IMSS y Sindicato acuerdo para implementar medidas de protección al personal durante contingencia por COVID-19. http://www.imss.gob.mx/prensa/ archivo/202003/138

Irby, D.M., \& Cooke, M. (2010). Calls for reform of medical education by the Carnegie Foundation for the Advancement of Teaching: 1910 and 2010, Academic Medicine, 85(2): 220-227. https://doi/10.1097/ ACM.0b013e3181c88449

Kebede, S., \& Pronovost, P. (2015). It is time to reinvent the wheels of medical training. Academic Medicine, 90(2), 126. https://doi/10.1097/ ACM.0000000000000600

Kepes, S., \& Andrew A. Evidence-based management and the trustworthiness of our cumulative scientific knowledge: Implications for teaching, research, and practice. Academy of Management Learning \& Education, 13(3), 446-466. https://doi/10.5465/amle.2013.0193

Mendoza, H., Méndez, J. F., \& Torruco, U. (2012). Aprendizaje basado en problemas $(\mathrm{ABP})$ en educación médica: sugerencias para ser un tutor efectivo. Investigación en Educación Médica, 1(4), 235-237. http://www. scielo.org.mx/pdf/iem/v1n4/v1n4a11.pdf

Monje, C. (2011). Metodología de la investigación cuantitativa y cualitativa. Guía didáctica. Universidad Surcolombiana. https://www.uv.mx/rmipe/ files/2017/02/Guia-didactica-metodologia-de-la-investigacion.pdf 
Moya, P., \& Williams, C. (2016). Efecto del Aula Volteada en el rendimiento académico: Estudio comparativo basado en el resultado del rendimiento académico con metodología Aula Volteada y Clase Tradicional para la asignatura de Salud Pública. R.E.C.S. 13(1), 15-20. https://revistas.psi.unc. edu.ar/index.php/revistaEF/article/view/22736

Muñoz, J. M., \& Maldonado, T. (2011). Aprendizaje con base en proyectos para desarrollar capacidades de problematización en educación superior. Actualidades Investigativas en Educación, 11(1),1409-4703. https://www. redalyc.org/articulo.oa?id=44718060014

Muñoz, J.M. (2012). Experiencia metacompleja para la construcción de un modelo de diagnóstico médico por estudiantes. FEM: Revista de la Fundación Educación Médica, 15(2), 89-94. http://scielo.isciii.es/pdf/edu/ v15n2/original1.pdf

Muñoz, J.M., Córdova, J., \& Priego, H. (2012). Dificultades y facilidades para el desarrollo de un proceso de innovación educativa con base en las Tecnologías de la Información y Comunicación (TIC). Formación Universitaria, 5(1), 3-12. https://doi/10.4067/S0718-50062012000100002

Muñoz, J.M., Maldonado, T., \& Bello, J. (2014). Desarrollo de proyectos para la formación de la competencia intercultural por estudiantes de medicina. FEM: Revista de la Fundación Educación Médica, 17(3), 161-169. http://scielo.isciii.es/pdf/fem/v17n3/original4.pdf

Muñoz, J.M., Maldonado, T., \& Olivares, A. (2014). Formación por competencia en educación médica: Capacidades y transgresiones necesarias. Perspectivas Docentes, 55, 5-10. http://revistas.ujat.mx/index.php/ perspectivas/article/view/1263/1013

Muñoz, J.M., \& Maldonado, T. (2017). Barriers to knowledge mobilization in learning to take evidenced-based clinical decisions: a descriptive longitudinal research. Perspectivas Docentes, 61, 28-35. https://doi. org/10.19136/pd.a0n61.1857 
Muñoz, J. M., Ramírez, J., Hernández, K., \& Córdova, J. A. (2020). Aprendizaje de estrategias para aplicación de ciencia en la educación médica. Formación Universitaria, en prensa.

Ortega, M., \& Cayoela, A. (2002). Medicina basada en la evidencia: una crítica filosófica a su aplicación en atención primaria. Revista Española de Salud Pública, 76(2), 115-120. http://scielo.isciii.es/pdf/resp/v76n2/a05v76n2.pdf

Organización Mundial de la Salud [OMS]. (2020a). ¿Cómo define la OMS la salud? https://www.who.int/es/about/who-we-are/frequently-askedquestions

Organización Mundial de la Salud [OMS]. (2020b). Determinantes sociales de la salud. https://www.who.int/social_determinants/es/

Pershing, S., \& Fuchs, V. R. (2013). Restructuring medical education to meet current and future health care needs. Academic Medicine, 88(12), 17981801. https://doi/10.1097/ACM.0000000000000020

Plá, S. (2018). Calidad educativa. Historia de una política para las desigualdades. ISSUE.

Rodríguez, R. (2014). Retos de las ciencias básicas en la educación médica. Gaceta Médica de México, 150(3), 358-360. https://www.anmm.org.mx/ GMM/2014/s3/GMM_150_2014_S3_358-360.pdf

Rojo, N., \& García, R. (2000). Sociología y salud: Reflexiones para la acción. Revista Cubana de Salud Pública, 26(2), 91-100. http://scielo.sld.cu/pdf/ rcsp/v26n2/spu03200.pdf

Román, J. A. (26 de abril de 2020). Universidades no estaban listas para clases en línea "de golpe": UAQ. La Jornada en línea. Recuperado de shorturl.at/gswOR

Román, J.A. (30 de abril de 2020). El 67.3\% de alumnos de la UNAM no logra adaptarse a clases virtuales. La Jornada en línea. Recuperado de shorturl.at/cizMS 
Rosas, M., \& Borrayo, G. (2018). Impacto de los nuevos criterios para diagnóstico y tratamiento de la hipertensión arterial sistémica sugeridos por la American College of Cardiology/American Heart Association. Gaceta Medica de Mexico, 154(6), 633-637. https://www.anmm.org.mx/GMM/2018/ n6/GMM_6_18_633-637.pdf

Ruiz, E. (2020). La práctica docente universitaria en ambientes de educación a distancia: Tensiones y experiencias de cambio. En Educación y pandemia: Una visión académica (pp. 109-114). ISSUE-UNAM.

Russell, B. (1959). Bertrand Russell: Un mensaje para el futuro. [Video]. YouTube. https://www.youtube.com/watch?v=eQYkqUlsIq0

Sagástegui, D. (2004). Una apuesta por la cultura: el aprendizaje situado. Revista Electrónica Sinéctica, 24, 30-39. https://www.redalyc.org/ pdf/998/99815918005.pdf

Sánchez, M. (2012). Educación médica basada en evidencias: ¿Ser o no ser? Investigación en Educación Médica, 1(2), 82-89. https://www.elsevier.es/esrevista-investigacion-educacion-medica-343-pdf-X2007505712427039

Secretaría de Salud. (14 de noviembre de 2016). Emite la Secretaría de Salud emergencia epidemiológica por diabetes mellitus y obesidad. Recuperado de shorturl.at/dhQW8

Seely, J., Collins, A., \& Dorguit, P. (1989). Situated cognition and the culture of learning. Educational Researcher, 18(1), 32-42. Recuperado de shorturl.at/ rwDX5

Tabung, F. K., Smith, S. A., Chavarro, J. E., Fung, T. T., Hu, F. B., Willett, W. C., \& Giovannucci, E. L. (2017). An empirical dietary inflammatory pattern score enhances prediction of circulating inflammatory biomarkers in adults. The Journal of Nutrition, 147(8), 1567-1577. https://doi.org/10.3945/ jn.117.248377

Torres, E., \& Osuna, C. (2011). Reprobación en las carreras del área de ciencias de la salud de la Universidad Autónoma de Baja California, México. Educación y Humanismo 13(21), 34-50. http://revistas.unisimon.edu.co/ index.php/educacion/article/view/2262 
Universidad Juárez Autónoma de Tabasco [UJAT]. (2020). Licenciatura en médico cirujano. Disponible en http://www.ujat.mx/285

Usagui, E. (2005). Durkhein: Conflicto y educación. Espacio y Tiempo: Revista de Ciencias Humanas, 19, 165-182. http://institucional.us.es/revistas/ cuestiones/17/art_17.pdf

Vargas, B., González, V., Orozco, C., Reyes, I., Santander, J., \& Pinedo, J. (2020). Rediseño e implementación de un curso de psicología médica desde metodología docente presencial, a una semipresencial. ARS MEDICA Revista de Ciencias Médicas, 45(2), 8-13. https://doi.org/10.11565/arsmed. v45i2.1624

Vergara, H. S. G., \& Carrillo, J. Q. (2018). Diseño e implementación de una propuesta didáctica por medio de la utilización de herramientas virtuales para el aprendizaje de inmunología en enfermedad periodontal. Acta Odontológica Colombiana, 8(2), 59-71. https://doi.org/10.15446/aoc. v8n2.73886

Vicedo, A. (2002). Abraham Flexner, pionero de la educación médica. Educación Médica Superior, 16(2), 156-163. http://scielo.sld.cu/pdf/ems/ v16n2/ems10202.pdf

Watmough S.D., \& O’Sullivan H. (2010). Graduates from a reformed undergraduate medical curriculum based on Tomorrow's Doctors evaluate the effectiveness of their curriculum six years after graduation through interviews. BMC Medical Education, 10. https://doi. org/10.1186/1472-6920-10-65

Willett, W., Rockström, J., Loken, B., Springmann, M., Lang, T., Vermeulen, S., Garnett, T., Tilman, D., DeClerck, F., Wood, A., Jonell, M., Clark, M., Gordon, L. J., Fanzo, J., Hawkes, C., Zurayk, R., Rivera, J. A., De Vries, W., Majele, L., Afshin, A., \& Murray, C. (2019). Food in the Anthropocene: the EAT-Lancet Commission on healthy diets from sustainable food systems. The Lancet, 393(10170), 447-492. https://doi.org/10.1016/S01406736(18)31788-4 